\title{
Psychometric Evidence of the Italian Anxiety Sensitivity Index-3 (ASI-3) in Patients with Anxiety Disorders and Psychosis
}

\author{
Andrea Pozza ${ }^{1,2, *}$, Davide Dèttore ${ }^{2,3}$ \\ ${ }^{1}$ Department of Experimental and Clinical Medicine, University of Florence, Italy \\ ${ }^{2}$ Miller Institute for Behavioural and Cognitive Psychotherapy, Genoa, Italy \\ ${ }^{3}$ Department of Health Sciences, University of Florence, Italy \\ *Corresponding author: apsycho@hotmail.it
}

Received March 11, 2015; Revised March 23, 2015; Accepted March 27, 2015

\begin{abstract}
The Anxiety Sensitivity Index-3 (ASI-3) is an 18-item measure, recently developed to assess AS, based, consisting of Physical Concerns (eg, the belief that palpitations lead to a cardiac arrest), Social Concerns (eg, the belief that publicly observable anxiety reactions will elicit social refusal), and Cognitive Concerns (eg, the belief that cognitive difficulties lead to insanity). In the Italian context there is a lack of a validated measure of AS, to date.The current study assessed factor structure, reliability, and validity of the Italian ASI-3 in large community and clinical samples. The Italian ASI-3, the Beck Depression Inventory-II, and the State and Trait Anxiety Inventory-trait subscale were administered to 547 community individuals and 146 patients with a primary panic disorder (PD) (n= $30)$, generalized anxiety disorder (GAD) $(n=33)$, social phobia $(\mathrm{SPh})(\mathrm{n}=25)$, or psychosis (PSY) $(\mathrm{n}=28)$. Exploratory and confirmatory factor analyses supported the three dimensions of AS in the community sample. All the ASI-3 dimensions showed good to excellent internal consistency, and moderate correlations with depression and trait anxiety measures. Physical Concerns discriminated PD patients from community individuals and the other clinical groups. Social Concerns discriminated SPh patients from community individuals and the other clinical groups. Patients with anxiety disorders had comparable scores on Cognitive Concerns and higher than community individuals. Patients with GAD endorsed higher scores than patients with PSY, specifically. Theoretical explanations, implication for research, and limitations are discussed.
\end{abstract}

Keywords: anxiety sensitivity, anxiety disorders, psychosis

Cite This Article: Andrea Pozza, and Davide Dèttore, "Psychometric Evidence of the Italian Anxiety Sensitivity Index-3 (ASI-3) in Patients with Anxiety Disorders and Psychosis.” Research in Psychology and Behavioral Sciences, vol. 3, no. 1 (2015): 7-17. doi: 10.12691/rpbs-3-1-3.

\section{Introduction}

\subsection{Construct and Measurement of Anxiety Sensitivity}

Anxiety Sensitivity (AS) is defined as an individual difference, that refers to the fear of arousal-related sensations [1]. AS is believed to arise from the combination of genetic vulnerability factor [1] and learning experiences that result in the acquisition of beliefs about potential harmful effects of autonomic arousal [1].

The first, most commonly used measure of AS is the Anxiety Sensitivity Index [ASI; 2]. Based on the first model of the construct [1], this tool was originally developed as unidimensional measure of AS. However, the unitary nature of AS construct has raised some criticism. Modern conceptualizations suggested a hierarchical multidimensional structure with a superordinate factor (the general AS) and three lower-order factors (Physical Concerns, Social Concerns, and Cognitive Concerns) [3]. High scores on the Physical Concerns factor denote worry about the physical health consequences of arousal sensations (eg, palpitations leading to a heart attack). High social concerns results from beliefs that publicly observable anxiety symptoms might lead to social rejection. Finally, high cognitive concerns are associated with worries that psychological symptoms, such as concentration difficulties, might lead to cognitive consequences such as mental incapacitation [3].

Factor analyses of the ASI did not support the unidimensional structure of AS [4]. Subsequent analyses of the psychometric properties of the ASI, and the theoretical work for refining the construct itself, suggested a multidimensional factor structure of the AS [4]. Indeed, the ASI included a Physical Concerns factor composed of eight items while the Social and Cognitive factors were made up of four items each. It has been argued that this might limit reliability of these two factors, and fail to adequately index the Social and Cognitive Concerns constructs [5].

The assessment of dimensionality in non-English speakers revealed a different number of factors, ranging from a single dimension [6] to a four-factor structure [7]. 
To better assess the lower-order AS dimensions, Taylor and Cox developed a 36-item revised scale, the Anxiety Sensitivity Index-revised [ASI-R; 8]. However, research on factor solutions has also provided inconsistent findings [9].

\subsection{Psychometric Evidence for the Anxiety Sensitivity Index-3 (ASI-3)}

\subsubsection{Factor Structure}

In a recent attempt to overcome conceptual and psychometric shortcomings of previous studies on the AS construct and its measures, Taylor and colleagues [5] developed the Anxiety Sensitivity Index-3 (ASI-3), an 18item self-report measure of AS. The measure was conceived as a multidimensional measure of AS. The instrument incorporated six items from the ASI-R (with one to two overlapping items each of the ASI-3's 6-item subscales), that best represented each of the three most common dimensions observed in previous research on AS: Physical, Social, and Cognitive Concerns [5]. Taylor and colleagues [5] assessed the three-factor structure of the measure using exploratory and confirmatory procedures in large samples of undergraduates from the United States and Canada ( $\mathrm{N}=2361)$. In this study, the three-factor solution was replicated in large samples of Non-English speaking undergraduates, specifically from France, Mexico, the Netherlands, and Spain [5]. The three-factor solution consisted of a Physical Concerns (eg, "It scares me when my heart beats rapidly”), a Social Concerns (eg, "It is important for me not to appear anxious"), and a Cognitive Concerns dimension (eg, "When I cannot keep my mind on a task, I worry that I might be going crazy”). The authors reported that the ASI-3 had a more stable factor structure than the ASI-R, and had improved psychometric properties, specifically higher reliability and construct validity, than the original ASI. In contrast to the ASI-R, the internal structure of the ASI-3 was stable across samples from different countries (Taylor et al., 2007).

\subsubsection{Specificity of the ASI-3 Across Anxiety Disorders and Other Disorders}

The lower-order AS dimensions measured by the ASI-3 seemed to be of different impact in the development and maintenance of anxiety disorders, as each dimension might be associated with thematically related psychopathology [5].

In the original validation study Taylor et al. [5] reported that Physical Concerns clearly discriminated patients with panic disorder (with or without agoraphobia) (PD) from those with other anxiety disorders, specifically generalized anxiety disorder (GAD), and social phobia (SPh). Social Concerns were specific to patients with SPh relative to those with the other three clinical groups. Patients with GAD or PD had the highest scores on the Cognitive Concerns dimension compared to the other two groups. Although the study conducted by Taylor et al. [5] provided evidence on the psychometric properties of the ASI-3, some limitations should be considered. First, both for the construction and validation of the ASI-3 were used data almost exclusively derived from undergraduate samples (not community samples), which could have limited heterogeneity in a variety of characteristics including age, occupational status, and education.

In a large clinical sample [10], the Physical Concerns dimension showed the highest correlation (moderate) with scales of body vigilance compared to the other dimensions. The Social Concerns dimension showed the highest correlation with a measure of fear of negative evaluation, and the correlation was moderate also for the Cognitive Concerns dimension. The Cognitive Concerns dimension had the highest correlation with depression and trait anxiety measures, despite correlations with these constructs were moderate for all the ASI-3 dimensions. In addition, the authors assessed known-groups validity, and reported that patients with PD had higher levels on the Physical Concerns dimension compared to patients with other anxiety disorders or those with disorders other than anxiety [10]. However, in this study patients with a diagnosis of other anxiety disorders (eg, SPh, GAD) were undersampled, and this limitation prevented to test for differences on the ASI-3 dimension scores between those disorders.

Wheaton et al. [11] investigated the specificity of the ASI-3 dimensions across an undergraduate control sample $(\mathrm{N}=315)$, and a large clinical sample $(\mathrm{N}=506)$ consisting of six diagnostic subgroups (patients with PD, OCD, SPh, GAD, health anxiety, or specific phobias). The Physical Concerns dimension strongly correlated with health anxiety, PD and worry measures, the Social Concerns dimension with social anxiety scales, and the Cognitive Concerns dimension with worry, PD and social anxiety measures. The ASI-3 total scores was significantly higher in each patient subgroup compared to the control sample [11]. Among the patient subgroups, the only significant difference, which emerged was that individuals with PD had significantly higher levels on the ASI-3 total than OCD patients [11]. With regard to the ASI-3 dimensions, patients diagnosed with $\mathrm{SPh}$ reported significantly higher scores than each of the other patient groups on the Social Concerns dimension [11]. The Panic Disorder subgroup scored significantly higher on the Physical Concerns dimension than the OCD, the GAD, and the SPh subgroup, but not than the Health Anxiety group [11]. All the patient subgroups scored significantly higher on the Cognitive Concerns dimension than the control sample, apart from the specific phobia subgroup, and patients with PD or GAD scored significantly higher than those with specific phobia [11].

There is still a lack of research on AS in patients with psychosis (PSY). To our knowledge, only a recent study assessed the AS dimensions in PSY patients using the ASI-3 [12]. Findings showed that PSY patients had significantly higher levels of AS than healthy controls in all the three ASI-3 dimensions [12]. However, the authors did not compare the scores of PSY patients to those of patients with primary anxiety disorders.

\subsection{Rationale for the Study}

In the original validation study, Taylor et al. [5] advocated the need for further research on factor structure in non-English speaking samples. Psychometric properties of the ASI-3 have not been yet assessed in Italian samples. A psychometric evaluation of an Italian ASI-3 appears important, since neither the original ASI [2] or the ASI-R 
[8] have been validated in Italy, and to date a measure of known psychometric properties to assess AS is not available for research and practice in healthcare settings.

In a recent meta-analysis, Naragon-Gainey [13] found that PD and agoraphobia were most closely related to both Physical and Cognitive Concerns, GAD to Cognitive and Social Concerns, whereas depression, $\mathrm{SPh}$, were all more moderately related to general AS, with depression specifically related to Cognitive Concerns and $\mathrm{SPh}$ to Social Concerns.

To our knowledge, only one study examined AS dimensions in patients with PSY using the ASI-3 [12]. The rationale for studying AS in PSY might be that anxiety disorders, and specifically social anxiety, appear to be relatively common among PSY patients. For example, Cosoff and Hafner [14] examined one hundred PSY inpatients, reporting that the proportion of inpatients with an anxiety disorder was relatively high (approximately 45\%), and $\mathrm{SPh}$ was the most common (17\%). Thus, it could be hypothesized that SPh and PSY may share common psychological processes [eg, 15], since fear of negative evaluation, for example, could play a role as a cognitive vulnerability underlying development or maintenance of PSY.

\subsection{Objectives and Hypotheses}

The objectives of the current study were to:

1. assess factorial validity and reliability of the Italian the ASI-3 in a community sample of Italian-speaking adult individuals (study 1 );

2. examine its the Italian ASI-3 with trait anxiety and depression measures in a community sample (study 2). Consistent with previous findings [eg, 10], we expected that (a) ASI-3 total would correlate moderately with trait anxiety and depression to a similar extent. We also expected that (b) Physical Concerns scores would correlate moderately with trait anxiety, and would correlate more strongly with trait anxiety compared to depression scores; (c) Social Concerns scores would correlate moderately with trait anxiety, and would correlate more strongly with trait anxiety compared to depression; (d) Cognitive Concerns scores would correlate moderately with both trait anxiety and depression scores.

3. investigate criterion-related validity. Specifically, we examined whether the ASI-3 scores would differ across community individuals, patients with a primary diagnosis of PD, GAD, SPh or PSY (study 3 ). We hypothesized that (a) clinical groups would have higher ASI-3 total scores than the community subsample. Consistent with previous evidence [eg, 12] we hypothesized that (b) the PD group would have higher Physical Concerns scores compared to the other groups, (c) the other clinical groups would have higher Physical Concerns scores than would the community subsample. We also expected that (d) the $\mathrm{SPh}$ group would have higher Social Concerns scores than would the other groups, (e) the other clinical group would have higher Social Concerns scores than the community subsample. Finally, given inconsistency of findings across studies about the specificity of Cognitive Concerns across anxiety disorders, we did not have an a priori hypothesis about this relation.

\section{Method}

\subsection{Participants}

For the current study, a community and a clinical convenience sample were recruited.

\subsubsection{Community Sample}

The questionnaires were administered to a total of 552 community (non-clinical) participants. Of these, five $(0.90 \%$ of the total community sample) were eliminated due to missing data. The final total community sample consisted of 547 Italian-speaking adults (age $\geq 16$ years, $65 \%$ women and $35 \%$ men). Individuals were recruited in a variety of public settings in several cities located in the Northern, Mid or Southern Italy.

The mean age was 45.92 years $(\mathrm{SD}=15.53$, range $=16$ 86). Mean years of education were 13.72 ( $\mathrm{SD}=4.13)$. All participants were white. Marital status was $44.40 \%$ single, $42.40 \%$ married or cohabitating, $10.10 \%$ separated or divorced, and $3.10 \%$ widowed. Sixty-two percent of the sample had a full- or part-time job, $12.10 \%$ were undergraduates, $6.60 \%$ unemployed, $16.40 \%$ retired, or $2.80 \%$ housewives.

In the community sample, participants were unscreened in order to obtain a more representative sample of the Italian community population.

\subsubsection{Clinical Sample}

Patients of the clinical sample were consecutive referrals to three outpatient centres for secondary mental health care.

Patients were included if they received a primary diagnosis of one of the following psychiatric disorders: panic disorder with or without agoraphobia (PD), generalized anxiety disorder (GAD), social phobia (SPh), or psychosis (PSY). Primary diagnoses were defined according to the following criteria: (a) the principal psychiatric problem in terms of severity and impairment, (b) the patient's primary source of distress, and (c) as the disorder for which the patient was seeking treatment.

Overall, exclusion criteria were: diagnosis of a bipolar disorder, having received cognitive-behavioural therapy during the past six weeks, mental retardation problems, and neurological conditions. To examine the criterionrelated validity of AS dimensions in patients with a primary diagnosis of an anxiety disorder without presenting with any other concurrent targeted anxiety disorder (or a depressive disorder) would be a better approach to investigate the specificity of AS dimensions to anxiety disorders in terms of internal validity. However, as suggested by some authors [16], such an approach could lack external validity since patients selected using this criterion would not be representative of populations of referrals in secondary care settings. Indeed, a key-point in establishing the concurrent validity of the AS dimensions across anxiety disorders is that approximately $50 \%$ of the patients with a principal anxiety disorder have at least one comorbid anxiety disorder at the time of assessment [16]. 
One hundred-sixty patients were recruited. Of those, 14 were excluded during enrolment because the target diagnosis was not assessed as primary by the two independent diagnosticians (eg, patients with bipolar disorder and secondary GAD).

The final total clinical sample consisted of 146 treatment-seeking adult patients. No missing data were found for this sample. Mean age was 48.53 years (SD= 13.94, range 18-83), and $49 \%$ were females. All participants were white. Mean years of education were 11.90 ( $\mathrm{SD}=4.44)$. Marital status was $40.60 \%$ single, $32.30 \%$ married or cohabitating, $21.80 \%$ separated or divorced, and $5.20 \%$ widowed. Fourty-five percent of the sample had a full- or part-time job, $4.20 \%$ were undergraduates, $17.70 \%$ unemployed, $26.00 \%$ retired, and $6.30 \%$ housewives.

In the clinical sample primary diagnoses were $\mathrm{PD}(\mathrm{n}=$ 30), GAD ( $n=33)$, SPh $(n=25)$, and PSY $(n=28)$.

Twenty-six percent of the clinical sample had a comorbid Axis I disorder according to the DSM-IV-TR (American Psychiatric Association, 2000). Of those patients $20.80 \%$ had a comorbid depressive disorder (major depressive disorder, dysthymic disorder or recurrent depression), and 6.30\% had a comorbid GAD. Twenty-four percent of the total clinical sample had a comorbid Personality Disorder (CPD) according to the DSM-IV-TR. Of those patients, $13.50 \%$ had a Borderline, $6.30 \%$ a Dependent, $2.10 \%$ an Istrionic, and $1.0 \%$ Obsessive-Compulsive or Narcissistic CPD.

\subsection{Measures}

Participants, both non-clinical and clinical, completed a questionnaire on socio-demographic information and a packet of three self-report clinical scales, including the ASI-3, the State-Trait Anxiety Inventory-Y form-trait subscale [STAI-Y; 17], and the Beck Depression Inventory-II [BDI-II; 18].

\subsubsection{Anxiety Sensitivity Index-3 (ASI-3)}

Subscale scores can be derived for the three dimensions, where higher scores are associated with greater AS. Respondents are asked to indicate their strength of endorsement for each item on a 5-point Likert scale $(0=$ "very little"; 4= "very much"). The ASI-3 and its subscales have shown good to very good internal consistency in non-clinical and clinical samples from five countries (alphas range $=$.73-.91) [5].

Before proceeding to the translation process of the measure, permission was obtained by the author who developed the scale (Prof. Steven Taylor). The translation process of the ASI-3 into Italian was undertaken using a protocol conforming to international standards [19]. This process included a forward and a backward translation. The forward translation was conducted by a native Italianspeaking clinical psychologist with excellent fluency in English, and checked by three Italian professional translators. The forward translator discussed the translation in consultation meetings with the professional translators. Subsequently, this version was translated back to English by a bilingual professional translator who was blind to the original ASI-3. The back translation was then compared with the original ASI-3, and discussed by the forwardtranslator with the backtranslator in a consensus meeting, which generated the final Italian version of the ASI-3. After translating the questionnaire, ten community Italian adults were administered with this pilot version of the Italian ASI-3, and interviews were conducted by a psychologist in order to check for semantic equivalence, comprehensibility, and content validity. Since this version appeared to be valid, it was used for the study.

\subsubsection{State-Trait Anxiety Inventory-Y form (STAI-Y- trait subscale)}

The STAI-Y-trait subscale was used to measure trait anxiety symptoms. The STAI-Y is a 20 -item self-report tool. Each item is rated on a 4-point intensity Likert scale with higher scores denoting higher levels of trait anxiety. In the current study internal consistency of the STAI-Y was very good for the total community sample (Cronbach's alpha= .89), and excellent for the total clinical sample $($ alpha $=.90)$.

\subsubsection{Beck Depression Inventory-II (BDI-II)}

The BDI-II was used to assess depressive symptoms. It is a 21-item self-reporting inventory rating the severity of depressive symptoms. Items are rated from 0 to 3 , and the total score ranges from 0 to 63 . Higher scores denote higher levels of depression. In the current study alpha was excellent for both the total community sample (alpha= .90) and the total clinical sample (alpha= .91).

\subsection{Procedure}

Data collection was carried out from January to November 2014 for both the community and the clinical sample. All the participants completed the questionnaires individually or in small groups. All the participants recruited for the study, both in the community and in the clinical samples, provided written informed consent to participate in the study after having received a description of the aims.

For the clinical sample, diagnoses were made through an unstructured clinical interview based on DSM-IV-TR criteria. For the anxiety disorders clinical sample, diagnoses were assigned independently by an experienced psychiatrist and a clinical psychologist. Despite no formal evaluation of between-assessors concordance on diagnosis was conducted, the assessors made biweekly or monthly clinical case meetings to establish consensus and monitor temporal stability of primary and secondary diagnoses.

\subsection{Data Analytic Plan}

\subsubsection{Factorial Validity And Reliability (study 1)}

For factorial validity, data from the community sample were randomly divided into two subsamples, such that responses from 298 participants (subsample 1) were used in Exploratory Factor Analysis (EFA), and responses from the remaining 249 participants (subsample 2) were used in Confirmatory Factor Analysis (CFA).

CFA was carried out using a structural equations modeling approach (Bollen, 1989). As in Taylor et al. (2007) and based on the results of the EFA in our study, we tested a model with three correlated factors. Subsequently, as Taylor et al. [5], we tested a two-factor model with a Physical Concerns factor and a combined factor consisting of items expected to load on the Social 
and Cognitive factors. In addition, we specified a singlefactor model in which all the 18 items load on a single factor.

To evaluate goodness of fit of the model to the data, the following indices recommended by $\mathrm{Hu}$ and Bentler [20] were used: the Adjusted Goodness-of-Fit Index (AGFI), the Goodness of Fit Index (GFI), the Bentler-Bonett Normed Fit Index (NFI), the Bollen's Relative Fit Index (RFI). For these indices, values close to 1 represent a good fit. In addition, the Root Mean Square Residual (RMR) was used as index of fit. For the RMR, values less than .08 represent acceptable fit, and values less than .05 represent good fit.

Reliability for the 18-item ASI-3 and for the three subscales was evaluated as internal consistency using Cronbach's alpha coefficients calculated on the total community sample ( $\mathrm{N}=547)$. Subsequently, internal consistency was assessed also for the total clinical sample $(\mathrm{N}=176)$. Reliability coefficients were evaluated according to Nunnally and Bernstein [21] (alpha> .70= acceptable, alpha $>.80=$ good, alpha $>.90=$ excellent). Further, reliability was verified as two-month-temporal stability using Pearson's correlation test-retest coefficients in a subsample $(n=58)$ of the community sample. In this subsample reliability was further tested computing intraclass correlation coefficients (ICC) by a two-way random effect model with absolute agreement between the measures.

\subsubsection{Concurrent and Discriminant Validity (study 2)}

To evaluate concurrent validity, we examined the correlations between the ASI-3 total and subscale scores and continuous measures of trait anxiety and depression in the total community sample $(\mathrm{N}=547)$. Concurrent validity of the three ASI-3 subscales was examined running bivariate correlations between the ASI-3 total and subscale scores, the STAI-Trait subscale, and the BDI-II scores. Significance levels were set at a Bonferronicorrected alpha value $(\mathrm{p}<.05 / 15)$ due to the number of correlations performed. In order to compare the bivariate correlation coefficients, the test for dependent coefficients recommended in Meng, Rosenthal, and Rubin [22] was applied.

Power calculations were run. For a medium effect size, $80 \%$ power, and significance set at the level described above, the required sample size for bivariate correlations was 155.

\subsubsection{Criterion-Related (Known Groups) Validity (study 3)}

To examine criterion-related validity of the ASI-3 and its subscales (study 3), we ran a series of one-way ANOVAs with post-hoc comparisons comparing the ASI3 total and subscale scores across the five clinical subsamples and a subsample $(n=30)$, randomly extracted from the total community sample. A Bonferroni correction for multiple comparisons adjustment was used $(\mathrm{p}<.003)$.

Power calculations were performed. For a medium ES, $80 \%$ power, and a significance set at the level described above, the required sample size was 96. Between-group effect sizes (ES) were estimated using the partial eta squared index. According to Cohen's recommendations [23], ES of $0.01,0.06,0.14$ were interpreted as small, medium, and large, respectively.
The EFA, reliability calculations, t-tests, ANOVAs, and bivariate correlations were conducted with SPSS software version 21.00. The CFA was performed with the AMOS software. Power calculations were performed using the GPower 3.1.7 software.

\section{Results}

\subsection{Item Distributional Properties and Scale Analyses}

To evaluate normality of items of the ASI-3, the inspection of curtosis and skewness indices for all the ASI-3 items was conducted. An absolute value on these indices falling out of the recommended range between -1 and +1 indicates a substantial deviance from normal distribution. Two of the 18 items ("When my throat feels tight, I worry that I could choke to death" and "When I feel "spacey" or spaced out I worry that I may be mentally ill”) showed a curtosis or skewness value out of the range, suggesting that data for these items were non-normally distributed. This observation was also supported by the inspection of the patterns of response frequencies, which revealed that the majority of participants $(85.20 \%$ for the first, and $86.40 \%$ for the latter item) endorsed these items as "very little" or "a little".

Independent samples t-test analyses showed that female community participants had higher scores than males on Physical Concerns $(t=-2.26, p=0.02)$, on Cognitive Concerns subscale $(\mathrm{t}=-2.35, \mathrm{p}=0.01)$, and on the total AS score $(\mathrm{t}=-2.19, \mathrm{p}=0.02)$. Despite females had higher scores also on Social Concerns subscale, this difference was non-significant $(\mathrm{t}=-0.90, \mathrm{p}=0.36)$.

Between-group differences were tested on the ASI-3 total and subscale scores as a function of age cohort. Results of one-way ANOVA showed a significant difference between young adults, adults, and older adults only on the Physical subscale $(\mathrm{F}=8.77, \mathrm{p}<.001)$. Post hoc comparisons suggested that older adults had significantly higher scores on the Physical Concerns subscale both than adults and young adults.

Descriptive statistics including means and standard deviations on the ASI-3 total and subscale scores for the total community sample as a function of gender are provided in Table 1.

Table 1. Means (standard deviations) on the ASI-3 total and subscale scores across gender for the total community sample $(N=547)$

\begin{tabular}{|c|c|c|}
\hline & Gender & \\
\hline & $\begin{array}{l}\text { Males } \\
(n=191) \\
M(S D)\end{array}$ & $\begin{array}{l}\text { Females } \\
(n=356) \\
M(S D)\end{array}$ \\
\hline \multicolumn{3}{|l|}{ ASI-3 } \\
\hline Total & $15.29(9.56)$ & 17.29 (11.15) \\
\hline Physical Concerns & $4.99(4.28)$ & $5.91(4.78)$ \\
\hline Social Concerns & 6.68 (3.79) & $7.00(4.18)$ \\
\hline Cognitive Concerns & $3.56(3.48)$ & $4.37(4.43)$ \\
\hline
\end{tabular}

\subsection{Study 1: Factorial Validity and Reliability of the ASI-3}

\subsubsection{Exploratory Factor Analysis (EFA)}

Prior to the extraction of factors, the assumptions required for EFA were tested. The Kaiser-Meyer-Olkin 
index of sampling adequacy (KMO) resulted 0.91, suggesting that the correlation matrix was appropriate for performing EFA, since it has been proposed that the KMO values should be equal or above |.60| in order to interpret satisfactorily an EFA solution [24]. The Bartlett's sphericity test (Bartlett, 1954) resulted significant, indicating that the data matrix was not an identity matrix $\left(\chi^{2}=2245.92, p=.000\right)$, thus being suitable for EFA.

Given non-normality of two of the ASI-3 items, factor extraction was carried out using the Principal Axis Factoring technique, following recommendations [24]. Using the criterion of eigenvalue greater than 1.0, three factors had eigenvalue over 1.0. The first factor had an eigenvalue of 7.93, the second of 1.92, and the third had an eigenvalue of 1.14 .

With regard to the number of factors retained, there is broad consensus in the literature, based on studies with Monte Carlo analyses, that retaining all factors with eigenvalues greater than 1.0 tends to lead to extract too many factors (i.e. overfactoring) [eg, 25]. Following recommendations [24], the number of factors to be extracted was determined by the visual inspection of the Scree test, which suggested the extraction of three factors.

Since the ASI-3 factors were moderately correlated with each other, as the Pearson's correlation coefficient values ranged between 0.52 and .65, the Promax oblique rotation technique was adopted.

These procedures evidenced a three-factor solution, which accounted for a total $53.60 \%$ of the variance in all the 18 items. The inspection of the factor loadings matrix did not reveal items which did not have salient loadings on any factor or items with salient loadings on more than one factor (i.e. crossloadings), as all the 18 items showed loading values greater than $|0.30|$ on a factor exclusively. A value of $|0.30|$ was chosen for the minimum of each item according to Tabachnik and Fidell's recommendations [24]. The first factor accounted for $41.65 \%$ of the total variance, and it was composed of all the six Physical Concern dimension items with very high factor loadings (all above 0.50 ). The second factor explained $8.25 \%$ of the variance, and consisted of the six items related to the Social Concern dimension, with five items with loadings greater than .50 and one item with a .48 loading. Finally, the third factor included all the six Cognitive Concern items, accounting for $3.68 \%$ of variance, with four items with loadings above .50 , one item with a .48 loading. One item had a smaller loading (.31) on this factor compared to the others. However, for this item loadings on the other factors were less than 20 .

Factor loadings and commonalities of each ASI-3 item for the three-factor solution are presented in Table 2.

Table 2. Factor loadings $\left(\lambda_{n}\right)$ and commonalities $\left(h^{2}\right)$ of the ASI-3 items on the three AS dimensions for subsample 1 ( $\left.n=298\right)$.

\begin{tabular}{|c|c|c|c|c|}
\hline & $\lambda_{1}$ & $\lambda_{2}$ & $\lambda_{3}$ & $h^{2}$ \\
\hline When I feel pain in my chest, I worry that I'm going to have a heart attack. & .96 & & & .72 \\
\hline When my chest feels tight, I get scared that I won't be able to breathe properly. & .81 & & & .71 \\
\hline When I notice my heart skipping a beat, I worry that there is something seriously wrong with me. & .80 & & & .61 \\
\hline When my throat feels tight, I worry that I could choke to death. & .67 & & & .55 \\
\hline It scares me when my heart beats rapidly. & .65 & & & .54 \\
\hline When my stomach is upset, I worry that I might be seriously ill. & .63 & & & .49 \\
\hline When I cannot keep my mind on a task, I worry that I might be going crazy. & & .81 & & .52 \\
\hline It scares me when I am unable to keep my mind on a task. & & .72 & & .54 \\
\hline When my thoughts seem to speed up, I worry that I might be going crazy. & & .70 & & .59 \\
\hline When I have trouble thinking clearly, I worry that there is something wrong with me. & & .67 & & .65 \\
\hline When I feel "spacey” or spaced out, I worry that I may be mentally ill. & & .54 & & .55 \\
\hline When my mind goes blank, I worry there is something terribly wrong with me. & & .48 & & .57 \\
\hline When I begin to sweat in a social situation, I fear people will think negatively of me. & & & .70 & .50 \\
\hline When I tremble in the presence of others, I fear what people might think of me. & & & .64 & .63 \\
\hline I worry that other people will notice my anxiety. & & & .63 & .61 \\
\hline It scares me when I blush in front of people. & & & .58 & .36 \\
\hline It is important for me not to appear nervous. & & & .48 & .33 \\
\hline I think it would be horrible for me to faint in public. & & & .31 & .22 \\
\hline
\end{tabular}

Note. Only loadings above |.30| are displayed.

\subsubsection{Confirmatory Factor Analysis (CFA)}

Prior to conduct the CFA, the assumption of multivariate normality was tested. Results of Kolmogorov-Smirnov tests indicated that the distribution of the items was significantly different from a normal distribution. In addition, curtosis and skewness values for 8 items resulted falling out of the recommended range for normality between +1 and -1 . Thus, the estimation method of Unweighted Least Squares was employed.

The three-factor model fit of the ASI-3, as it was found in the English validation study (Taylor et al., 2007) and in our study 1, was investigated. All the indices considered suggested good fit of the model to the data (GFI= .98; $\mathrm{AGFI}=.97 ; \mathrm{NFI}=.96 ; \mathrm{RFI}=.96 ; \mathrm{RMR}=.05)$.
As previously reported by Taylor et al. [5] and Wheaton et al. [11], a single-factor and a two-factor solutions were tested. A single-factor model was tested, in which all the 18 items of the ASI-3 loaded on a single factor. However, only one index of those considered showed an acceptable fit of the model to the data $(\mathrm{GFI}=.92)$, whereas the other indices indicated poor fit $(\mathrm{AGFI}=.90 ; \mathrm{NFI}=.88$; $\mathrm{RFI}=.87$; $\mathrm{RMR}=.10)$, suggesting that a model specifying a single factor of the ASI-3 should be rejected.

Subsequently, a two-factor model was tested, built on a Physical Concerns factor and a combined factor in which items from the Social and the Cognitive factors loaded on a single one. Despite most of the indices showed good fit $(\mathrm{GFI}=.97 ; \mathrm{AGFI}=.96 ; \mathrm{NFI}=.94 ; \mathrm{RF}=.94)$, one index suggested only acceptable fit $(\mathrm{RMR}=.07)$. 
Since the three-factor model showed improved goodness of fit in all the considered indices compared to single- and two-factor models, the results seemed to suggest that a three-factor solution had the best overall fit to the data, supporting the theoretical assumptions of the original version of the ASI-3 [5].

An overview of fit indices for the three ASI-3 models specified is provided in Table 3. The three-factor ASI-3 model is presented in Figure 1.

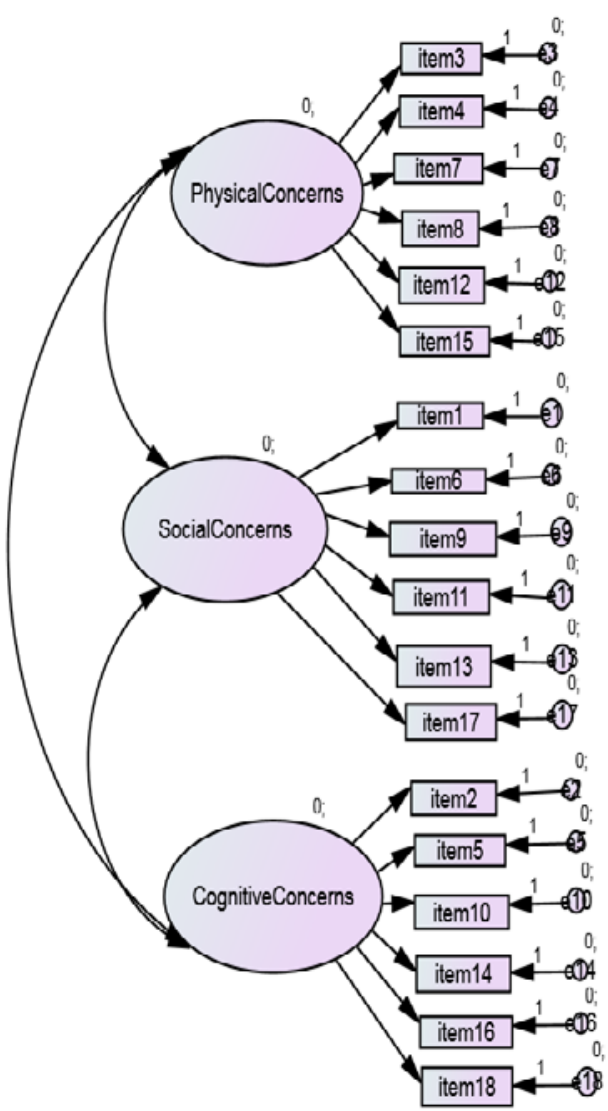

Figure 1. Confirmatory three-factor model of the ASI-3

Table 3. Fit indices of the Italian ASI-3 for a single-factor, two-factor, and the original three-factor models tested on subsample 2 of study 1 $(n=249)$

\begin{tabular}{lccccc}
\hline Model tested & GFI & AGFI & NFI & RFI & RMR \\
\hline One-factor model $^{\mathrm{a}}$ & .92 & .90 & .88 & .87 & .10 \\
Two-factor model $^{\mathrm{b}}$ & .97 & .96 & .94 & .94 & .07 \\
Three-factor original model $^{\mathrm{c}}$ & .98 & .97 & .96 & .96 & .05 \\
\hline
\end{tabular}

Note. $^{\text {a }}$ In the single-factor model all the 18 items of the ASI-3 load on a single factor. ${ }^{\mathrm{b}}$ The two-factor model consisted of a Physical Concerns factor and a combined Social/Cognitive Concerns factor as tested by Taylor et al. (2007). ${ }^{\mathrm{C}}$ The original model is built on the three-factor model tested in the original validation study (Taylor et al., 2007). Abbreviations. AGFI= Adjusted Goodness-of-Fit Index; GFI= Goodness of Fit Index; NFI= Bentler-Bonett Normed Fit Index; RFI= Bollen's Relative Fit Index; RMR= Root Mean Squared Residual.

\subsubsection{Reliability}

Reliability as internal consistency was analyzed for the total community sample $(\mathrm{N}=547)$ and for the total clinical sample $(\mathrm{N}=176)$, separately.

In the total community sample, the Cronbach's alpha estimate for the ASI-3 total score was 0.91 (range of corrected item-total correlations $=0.26-0.68$ ), suggesting excellent internal consistency. In addition, all the corrected item-total score correlations were greater than .20.
Internal consistency was very good for Physical Concerns (alpha $=0.88$ ) and Cognitive Concerns subscales (alpha $=0.86)$, whereas it was good for Social Concerns subscale (alpha $=0.77$ ).

In the total clinical sample, the Cronbach's alpha estimate for the ASI-3 total score was 0.93 (range of corrected item-total correlations $=0.35-0.72$ ), suggesting excellent internal consistency. Internal consistency was excellent for Physical Concerns $(\alpha=0.90)$, and very good for Cognitive Concerns $(\alpha=0.88)$, and Social Concerns subscales (alpha $=0.84)$.

On a subsample $(n=58)$ of the total community sample, two-month temporal stability was good only for the Social Concerns subscale since test-retest correlation was 0.71 , while it was modest for the total and the other subscale scores, since test-retest correlations were $0.67,0.66$, and 0.62 (all ps $<.001$ ), respectively. Temporal stability of the ASI-3 was also supported by the ICC, that was for the total score 0.79 (95\% CI: 0.63-0.88), for the Physical Concerns subscale 0.79 (95\% CI: 0.65-0.87), for the Social Concerns subscale 0.82 (95\% CI: 0.70-0.89), and for the Cognitive Concerns subscale 0.75 (95\% CI: 0.580.85).

\subsection{Study 2: Concurrent and Discriminant Validity}

Concurrent validity between the ASI-3 total and subscale scores and trait anxiety and depression was examined in the total community sample $(\mathrm{N}=547)$.

The ASI-3 total scores showed moderate bivariate correlations with both trait anxiety and depression scores (Pearson's r of 0.36 and 0.32, respectively. All ps<.003). Physical Concerns scores correlated moderately with trait anxiety (Pearson's $\mathrm{r}=0.30, \mathrm{p}<.003$ ) and weakly with depression scores (Pearson's $r=0.24, p<.003$ ). Social Concerns scores moderately correlated with trait anxiety (Pearson's $\mathrm{r}=0.36, \mathrm{p}<.003$ ) and weakly with depression scores (Pearson's $r=0.29, \mathrm{p}<.003$ ). Cognitive Concerns moderately correlated with both trait anxiety (Pearson's $\mathrm{r}=$ 0.52, $\mathrm{p}<.003$ ) and depression scores (Pearson's $\mathrm{r}=0.30$, $\mathrm{p}<.003)$.

Contrary to our predictions, the correlation of the Physical Concerns subscale scores with trait anxiety scores was not significantly higher than that observed with depression scores to establish discriminant validity $(\mathrm{z}=$ 1.07, $\mathrm{p}>.05)$. Moreover, for the Social Concerns subscale, the correlation of this subscale with trait anxiety scores to establish concurrent validity was not significantly higher than that observed with depression scores $(\mathrm{z}=1.30, \mathrm{p}>.05)$.

In line with our hypothesis concerning concurrent validity of general AS, the correlation of ASI-3 total scores with trait anxiety scores was not significantly different from that found with depression scores $(\mathrm{z}=0.74$, $\mathrm{p}>$.05). In addition, our prediction about concurrent validity of the Cognitive Concerns subscale, the correlation of this subscale scores with trait anxiety scores was not significantly different from that found with depression scores $(\mathrm{z}=0.00, \mathrm{p}>.05)$.

\subsection{Study 3: Criterion-related (Known Groups) Validity}

Known-groups validity was examined comparing on the ASI-3 total and subscale scores the PD, GAD, SPh, PSY 
groups and a subsample randomly extracted from the total community sample $(n=30)$. Non-significant Levene's tests suggested that the assumption of homogeneity of variances was not violated $(\mathrm{F}=2.10-0.75, \mathrm{p}=.59-.07)$. Results of one-way ANOVAs with LSD post-hoc comparisons showed that PD, GAD and SPh groups had significantly higher scores on the ASI-3 total scores than the community subsample and the PSY group $(F=7.99$, $\mathrm{p}<.001$ ). Between-group ES was large (partial $\eta^{2}=.25$ ) according to Cohen's recommendations (1988). The PD group had significantly higher scores on the Physical Concerns subscale than the community subsample and the GAD, SPh, or PSY groups $(F=7.53, p<.001)$. Betweengroup ES was large (partial $\eta^{2}=.24$ ). The $\mathrm{SPh}$ and the
GAD groups had significantly higher scores on the Social Concerns subscale compared to the community subsample; in addition, the SPh group had significantly higher scores than the PD, GAD, or PSY groups $(F=6.64, p<.001$, partial $\left.\eta^{2}=.22\right)$. The PD and GAD, and SPh groups had significantly higher scores on the Cognitive Concerns scores than the community subsample; in addition, the GAD group had significantly scores compared to the or PSY group $\left(\mathrm{F}=7.54, \mathrm{p}<.001\right.$, partial $\left.\eta^{2}=.24\right)$.

An overview of descriptive statistics on the ASI-3 total and subscale scores and post-hoc comparisons tests across the community subsample and the five clinical subsamples is provided in Table 4.

Table 4. Group means (standard deviations) and comparisons on the ASI-3 total and subscale scores across the community subsample, PD, GAD, SPh, and PSY subsamples

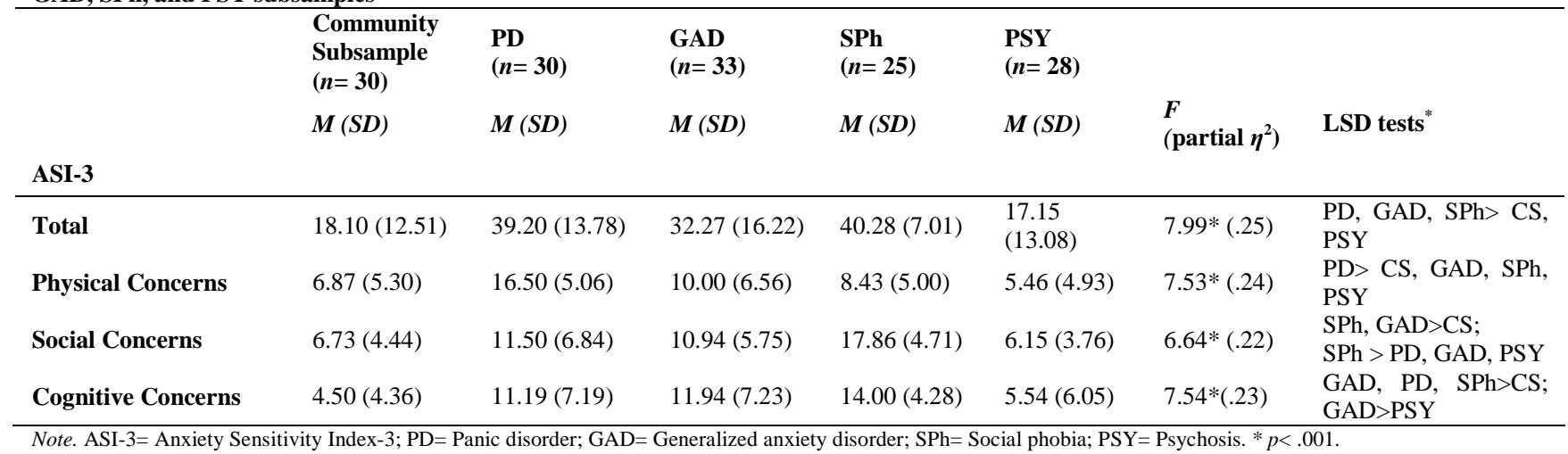

\section{Discussion}

\subsection{Synthesis of findings}

\subsubsection{Study 1: Factorial Validity and Reliability}

In the exploratory study we found support for a threefactor solution, which showed a clearly-defined structure as all the 18 items had salient loadings $\left(\lambda_{n}>|.30|\right)$ on a single factor exclusively, and the magnitude of the commonalities suggested that the three factors explained a substantial proportion of variance in all items. The three factors extracted resulted moderately correlated with each other, suggesting that they represent related but separate constructs of AS. The three-factor solution of the Italian ASI-3 appeared consistent with previous findings [eg, 5], either with non-clinical or clinical individuals [eg, 10]. Confirmatory factor procedures also supported a threefactor model according to all the fit indices considered. Thus, overall current findings provided further evidence for the stability of a three-factor structure of AS construct across countries.

The current results seemed to provide some support for reliability of the Italian ASI-3. The overall internal consistency resulted excellent for the ASI-3 both in the community and in the clinical samples. Internal consistency appeared very good or good for the three subscales in both the samples. Such findings appeared in line with results found in previous validation studies [5].

Since AS is believed to be a trait feature, examining temporal stability of the construct can be relevant. Results showed that using a quite large time interval (i.e. two months), temporal stability of the measure was adequate only for the Social Concerns subscale, whereas it was modest for the total, Physical, and Cognitive Concerns subscale scores.

\subsubsection{Study 2: Concurrent and Discriminant Validity}

For the current study depression and trait anxiety were used as constructs to investigate concurrent and discriminant validity of the ASI-3 total and subscales. Depression scores were used to test for concurrent validity with the ASI-3 total and the Cognitive Concerns subscale, and for discriminant validity with the Physical and Social Concerns subscales. Trait Anxiety scores were used to test for concurrent validity with the ASI-3 total and the three subscales.

Data indicated moderate correlations between the ASI-3 total scores and trait anxiety, which were in line with previous research in non-English speaking samples [eg, 28]. However, that study did not assess correlation between trait anxiety and the ASI-3 dimensions, specifically. Our study provided some evidence on the relations of AS dimensions to trait anxiety, suggesting that specific dimensions could be related to trait anxiety. All the three AS dimensions moderately correlated with trait anxiety, with Cognitive Concerns showing the highest pattern of correlation. Trait anxiety was conceptualized as the tendency to respond with fear to a wide range of stressors [17]. AS can be assumed to be a distinct construct from trait anxiety, since the former represents a more specific tendency to fearfully respond to the consequences (physical, social, or psychological) of one's own anxiety symptoms.

In the current study, evidence of concurrent validity was found also between AS dimensions and depression symptoms. Moderate relations between AS dimensions and depression were observed. However, only Cognitive Concerns appeared specifically related to depression 
compared to the other dimensions. These findings were consistent with both previous research using the original ASI [eg, 26] and the ASI-3 [eg, 10], and they suggested that the relationship between AS and depression might be specific to fear of mental incapacitation [29]. Mental incapacitation might amplify depressive symptoms, for example by catastrophic interpretations of concentration difficulties as signs of an impairing depressive condition [30]. This explanation is supported by recent longitudinal data from clinical samples [31], indicating that individuals with more severe depressive symptoms are more likely to experience impairment from nervousness and concentration difficulties, thus reinforcing concerns about mental incapacitation. However, it should be argued that the current results appeared in contrast with other findings reported with the ASI-3 by Osman et al. [32], which suggested that depression symptoms were more strongly related to Social than Cognitive Concerns.

Although a large body of research has predominantly supported the relation between the Cognitive Concerns component of AS and depression, it should be noted that other studies have produced conflicting results [33], and in some studies, both cross-sectional and prospective on nonclinical samples, Cognitive Concerns did not predict depressive symptoms [33]. In addition, some crosssectional studies have reported evidence of association between the Physical and Social components with depression [eg, 26], and prospective investigations showed that Physical Concerns significantly predicted increases in depression symptoms at one-year follow up [27].

\subsubsection{Study 3: Criterion-related Validity}

In line with our predictions, current data seemed to support the assumption that general AS is a marker specific to anxiety disorders, since all the patients with a primary anxiety disorder had significantly higher ASI-3 total scores compared to community individuals and AD or PSY patients. In addition, no significant difference was found between the anxiety disorder groups. These findings appeared consistent with evidence yielded by Taylor et al. [5] and with a meta-analysis on the studies using the ASI [34]. Current evidence appeared also in line with previous longitudinal research. For example, in a prospective study [35], AS has been found to predict the incidence of any anxiety disorder diagnosis at two-year follow-up in a large sample of young non-clinical individuals with no history of Axis I diagnoses at study entry. Thus, our data seemed to contrast with earlier AS conceptualizations as a vulnerability factor specific to PD [2]. However, the current results appeared in contrast with those reported by other studies using the ASI-3 [11], which showed that PD patients had higher levels of general AS compared to both non-clinical individuals and those with other anxiety disorders. Contrary to our predictions, PSY patients had not higher levels of general AS compared to community individuals.

Our findings suggested that PD patients had significantly higher Physical Concerns than both community and the other clinical groups. These results appeared consistent with previous evidence emerged with the ASI-3 [eg, 5], supporting the notion that Physical Concerns could be a vulnerability and maintenance factor specific to $\mathrm{PD}$, also in accordance with previous literature with the original ASI. For example, prospective studies showed that ASI scores predicted the development of spontaneous panic attacks among undergraduates [36]. Overall, these findings appeared to confirm the Clark's model of PD for case formulation [37], and supported the notion that Physical Concerns may be a specific target of cognitive-behavioural therapy (CBT) for PD.

Consistent with our predictions, Social Concerns resulted significantly higher among patients with SPh compared to both non-clinical subsample and the other clinical groups, suggesting that they could be a vulnerability factor specific to $\mathrm{SPh}$. These findings appeared in line with previous research using the ASI-3 [eg, 11], supporting the notion that case formulation and CBT for SPh should aim to target catastrophic beliefs underlying fear of negative evaluation.

Another result was that patients with PSY had comparable scores on the Social Concerns subscale to non-clinical individuals, suggesting that Social Concerns could not be relevant to PSY. However, this could be linked to the inclusion of both patients with PSY in an acute and a stable phase of the disorder. In effect, some research suggested that patients with PSY reported higher levels of social concerns during an acute than during a stable phase of the disorder [12].

With regard to Cognitive Concerns, in our study patients with any anxiety disorder endorsed significantly higher scores on the ASI-3 Cognitive Concerns subscale compared to non-clinical individuals. In addition, GAD patients, but not patients with other anxiety disorders, had significantly higher scores than PSY patients. These findings suggested that Cognitive Concerns could be a vulnerability factor common to anxiety disorders, and specifically to GAD. An explanation for these findings could be that in previous research GAD appeared associated to metacognitions about the harmful effects of persistent worry [38]. However, such results appeared in contrast with previous findings reported by Taylor et al. [5], which showed that Cognitive Concerns were more specific to both GAD and PD patients, and to the study by Wheaton et al. [11], in which Cognitive Concerns were common to all anxiety disorders, also including health anxiety and OCD.

In addition, in our study only patients with GAD had significantly higher scores on Cognitive Concerns compared to patients with PSY. Strong Cognitive Concerns could be a consequence of impairment related to psychotic negative symptoms, or to the awareness of cognitive deficits frequently associated to PSY. This explanation could take into account why the other anxiety disorder groups had not higher scores than patients with PSY. However, it should be noted the lack of significant differences between PSY patients and non-clinical individuals, which could be related to the fact that not all the PSY patients were on an acute phase of the disorder.

\subsection{Limitations and Implications for Research}

Findings of the current study should be considered in the context of some limitations. First, due to the lack of known psychometric properties on the original ASI in Italian samples, in the current study incremental validity of the ASI-3 was not evaluated in comparison with the ASI. 
Moreover, despite an attempt was made to assess concurrent/discriminant validity of the measure using trait anxiety and depression comparators, the relationship between the ASI-3 subscales and thematically related symptoms was not further investigated. Thus, future research should assess the use of such measures to improve knowledge on the Italian ASI-3 validity, for example testing the relation between the ASI-3 Social Concerns subscale and social anxiety measures, or between the ASI-3 Physical Concerns and body vigilance questionnaires.

In addition, due to the relatively small size of the total clinical sample, factor structure could not be assessed in individuals with a psychiatric diagnosis.

Our study lacked some diagnostic groups, which could be related to AS dimensions (eg, post-traumatic stress disorder or obsessive-compulsive disorder). For example, a recent study by Wheaton et al. [11] using the ASI-3 suggested that levels of AS dimensions can differ across OCD symptoms.

In addition, predictive validity of the ASI-3 still remains unexplored. Future research could test whether individuals endorsing higher scores on specific AS dimensions, would develop symptoms of thematically related anxiety disorders, as hypothesized by AS modern conceptualizations [eg, 5].

A further exploration on the specificity of AS dimensions in the maintenance of anxiety disorders could be the examination of responsiveness of the ASI-3 after CBT or other treatments targeting AS. In effect, the ASI-3 could be used in longitudinal research on risk factors for the development of anxiety symptoms, or it could be used as outcome in randomized controlled trials on CBT on processes driving symptom change of different anxiety disorders.

\section{Conclusions}

Overall, findings provided additional support for dimensionality of the AS construct in a non-English speaking population.

AS dimensions appeared to discriminate patients with thematically related psychopathology. Physical and Social Concerns resulted able to discriminate patients with PD and SPh respectively from non-clinical individuals and from patients with other psychiatric diagnoses. The Cognitive Concerns dimension appeared to be a cognitive vulnerability or maintenance factor common to all the anxiety disorders, specifically to GAD.

In conclusion, the ASI-3 seems to be a valid and reliable measure, which can be used in the context of assessment and treatment of anxiety disorders in both research and clinical practice.

\section{Conflict of Interest}

The authors have no conflict of interest to declare.

\section{References}

[1] Reiss S, McNally RJ. Expectancy model of fear. In S. Reiss \& R.R. Botzin (Eds.), Theoretical issues in behavior therapy (pp. 107121). San Diego, CA: Academic Press, 1985.
[2] Reiss S, Peterson RA, Gursky DM, McNally RJ. Anxiety sensitivity, anxiety frequency, and the prediction of fearfulness. Behav Res Ther 1986; 24: 1-8.

[3] Taylor SE. Anxiety sensitivity: Theory, research, and treatment of the fear of anxiety. Lawrence Erlbaum Associates Publishers: Mahwah, NJ, 1999.

[4] Deacon B, Abramowitz JS. Anxiety sensitivity and its dimensions across the anxiety disorders. J Anxiety Disord 2006; 20: 837-857.

[5] Taylor S, Cox BJ, Holaway RM, Stewart SH, Eng W, Arrindell WA, Zvolensky MJ, Deacon B, Abramowitz, JS, Sandin B, Coles M, Daly ES, Bouvard M. Robust dimensions of anxiety sensitivity: Development and initial validation of the Anxiety Sensitivity Index-3. Psychological Assessment 2007; 19: 176-188.

[6] Sandìn B, Chorot P, McNally RJ. Validation of the Spanish version of the Anxiety Sensitivity Index in a clinical sample. Behav Res Ther 1996; 34: 283-290.

[7] Maruta T, Yamate Ito K, Sato M, Iimori M, Kato M. Reliability and validity of the Japanese version of the Anxiety Sensitivity Index. Compr Psychiatry 2007; 48: 289-292.

[8] Taylor S, Cox BJ. (1998). An expanded Anxiety Sensitivity Index: Evidence for a hierarchic structure in a clinical sample. J Anxiety Disord 1998; 12: 463-483.

[9] Deacon BJ, Abramowitz JS, Woods CM, Tolin DF. The Anxiety Sensitivity Index-Revised: psychometric properties and factor structure in two nonclinical samples. Behav Res Ther 2003; 41: 1427-1449.

[10] Kemper CJ, Lutz J, Bähr T, Rüddel H, Hock M. Construct validity of the anxiety sensitivity index-3 in clinical samples. Assessment 2012; 19: 89-100.

[11] Wheaton MG, Deacon BJ, McGrath PB, Berman NC, Abramowitz JS. Dimensions of anxiety sensitivity in the anxiety disorders: Evaluation of the ASI-3. J Anxiety Disord 2012; 26: 401-408.

[12] Luzón O, Harrop C, Nolan F. Cognitive Processes during Acute Psychosis: The Role of Heightened Responsibility and Catastrophic Misinterpretations. Behavioural and Cognitive Psychotherapy 2009; 37: 357.

[13] Naragon-Gainey K. Meta-analysis of the relations of anxiety sensitivity to the depressive and anxiety disorders. Psychol Bull 2010; 136: 128.

[14] Cosoff SJ, Hafner RJ. The prevalence of co-morbid anxiety in schizophrenia, schizoaffective disorder and bipolar disorder. Austr N Z J Psychiatry 1998; 32: 67-72.

[15] Michail M, Birchwood M. Social anxiety disorder and shame cognitions in psychosis. Psychol Med 2013; 43: 133-142.

[16] Barlow DH. Anxiety and its disorders: The nature and treatment of anxiety and panic. New York: The Guilford Press, 2002.

[17] Spielberger CD. Manual for the State-Trait Anxiety Inventory. Palo Alto, CA: Consulting Psychologists Press, 1983.

[18] Beck AT, Steer RA, Brown GK. Beck Depression Inventory Second Edition Manual. San Antonio, TX: The Psychological Corporation Harcourt Brace Company, 1996.

[19] Behling O, Law KS. (Eds.). Translating questionnaires and other research instruments: Problems and solutions. Thousand Oaks: Sage, 2000.

[20] Hu LT, Bentler PM. Cutoff criteria for fit indexes in covariance structure analysis: Conventional criteria versus new alternatives. Structural Equation Modeling: A Multidisciplinary Journal 1999; 6: 1-55.

[21] Nunnally J, Bernstein I. Psychometric theory. New York: McGraw-Hill, 1994.

[22] Meng XL, Rosenthal R, Rubin DB. Comparing correlated correlation coefficients. Psychol Bull 1992; 111: 172-175.

[23] Cohen J. Statistical power analysis for the behavioral sciencies. Routledge: New York, 1998.

[24] Tabachnick BG, Fidell LS. Using multivariate statistics. New York: Harper Collins, 2001.

[25] Velicer WF, Jackson DN. Component analysis versus common factor analysis: Some issues in selecting an appropriate procedure. Multivariate Behavioral Research 1990; 25: 1-28.

[26] Rector NA, Szacun-Shimizu K, Leybman M. Anxiety sensitivity within the anxiety disorders: Disorder-specific sensitivities and depression comorbidity. Behav Res Ther 2007; 45: 1967-1975.

[27] Olthuis JV, Watt MC, Stewart SH. Anxiety Sensitivity Index (ASI-3) subscales predict unique variance in anxiety and depressive symptoms. J Anxiety Disord 2014; 28: 115-124. 
[28] Mantar A, Yemez B, Alkin T. The Validity and Reliability of the Turkish Version of the Anxiety Sensitvity Index-3. Turkish Journal of Psychiatry 2010; 21: 1-9.

[29] Taylor S, Koch WJ, Woody S, McLean P. Anxiety sensitivity and depression: how are they related? J Abnorm Psychol 1996; 105: 474.

[30] Cox BJ, Enns MW, Taylor S. The effect of rumination as a mediator of elevated anxiety sensitivity in major depression. Cognitive Therapy and Research 2001; 25: 525-534.

[31] Rosellini AJ, Fairholme CP, Brown TA. The temporal course of anxiety sensitivity in outpatients with anxiety and mood disorders: relationships with behavioral inhibition and depression. J Anxiety Disord 2011; 25: 615-621.

[32] Osman A, Gutierrez PM, Smith K, Fang Q, Lozano G, Devine A. The Anxiety Sensitivity Index-3: Analyses of Dimensions, Reliability Estimates, and Correlates in Nonclinical Samples. J Pers Assess 2010; 92: 45-52.
[33] Ho MHR, Auerbach RP, Jun HL, Abela JR, Zhu X, Yao S. Understanding anxiety sensitivity in the development of anxious and depressive symptoms. Cognitive Therapy and Research 2011; 35: 232-240.

[34] Olatunji BO, Wolitzky-Taylor KB. Anxiety sensitivity and the anxiety disorders: a meta-analytic review and synthesis. Psychol Bull 2009; 135: 974.

[35] Schmidt NB, Zvolensky MJ, Maner JK. Anxiety sensitivity prediction of panic attacks and Axis I pathology. J Psychiatr Res 2006; 40: 691-699.

[36] Plehn K, Peterson RA. Anxiety sensitivity as a predictor of the development of panic symptoms, panic attacks, and panic disorder: a prospective study. J Anxiety Disord 2002; 16: 455-474.

[37] Clark DM. A cognitive approach to panic. Behav Res Ther 1986; 24: 461-470.

[38] Wells A. The metacognitive model of GAD: assessment of metaworry and relationship with DSM-IV generalized anxiety disorder. Cognitive Therapy and Research 2005; 29: 107-121. 